\title{
Self-similarity of trailing vortices
}

David M. Birch ${ }^{1, \text { a) }}$

University of Surrey, Guildford, Surrey, UK, GU2 7XH

( Dated: 7 February 2012)

Trailing vortices have been repeatedly shown to exhibit a remarkably robust selfsimilarity independent of the Reynolds number and upstream boundary conditions. The collapse of the inner-scaled circulation profiles of a trailing vortex has even been previously demonstrated for the cases of highly unsteady and turbulent vortex systems, as well as for vortices which were incompletely developed. A number of factors which contribute to and may artificially promote this self-similarity are discussed. It is shown that the amplitude of vortex 'wandering' (or the random modulations in the vortex trajectory) observed in some experimental measurements are of sufficient amplitude to cause any arbitrary finite and axisymmetric flow structure to collapse with an idealized trailing vortex when scaled on inner parameters. It is further shown that, for the case of an incompletely developed wing-tip vortex, similarity in the outer core region may be an artefact of the rate of roll-up of the vortex sheet. Great care must therefore be taken when interpreting experimental measurements of vortex flows.

\footnotetext{
a)Electronic mail: d.birch@surrey.ac.uk
} 


\section{INTRODUCTION}

Vortex flows have historically been of significant interest, both because of their relevance to industrial and aeronautical applications as well as their fundamental importance to all turbulent flows. More recently, wing-tip vortices have received particular attention, owing to their contribution to aircraft fuel burn and atmospheric carbon emissions and their importance in determining the minimum aircraft separation distances that limit airport capacity.

While the mechanisms behind the formation and development of the trailing vortices behind wing-tips or vortex generators is already well understood (see, for example, the reviews of Widnall ${ }^{1}$, Spalart ${ }^{2}$ and $\mathrm{Kroo}^{3}$, and references cited therein), the role of turbulence and its effect on developing vortices is not as clear.

There is compelling evidence that turbulence plays only a passive role in vortex development. The radial profile of tangential velocity within the highly vortical inner core is nearly linear, so that the flow is expected to be dominated by viscosity, rapidly dissipating any turbulence occurring within that region. This 'inactive core' model is supported by a number of experimental studies at reasonably high Reynolds number, including those of Phillips and Graham ${ }^{4}$, Devenport et al. ${ }^{5}$ and Chow et al. ${ }^{6}$. On the other hand, some studies (such as those of Green and Acosta ${ }^{7}$, Sarpkaya ${ }^{8}$, Beninati and Marshall ${ }^{9}$ and Birch and Lee ${ }^{10}$, amongst others) have demonstrated very high levels of turbulence within a vortex core, suggesting dissipation rates inconsistent with the inactive core model.

Though it is possible that the persistence of turbulence within a vortex core stems from a combination of high turbulence levels in the generating boundary layers and insufficient streamwise development, it has also been suggested that these conflicting observations may be indicative of the presence of coherent structures transported within the vortex. The velocity statistics of Bandyopadhyay, Stead, and Ash ${ }^{11}$, for example, showed high levels of turbulent stresses within a vortex alongside evidence of local relaminarization, consistent with the existence of convected secondary structures within the vortex. Indeed, given that vortices and boundary layers are known to be analogous ${ }^{12,13}$, and there is evidence of transport within boundary layers by large, transient structures ${ }^{14}$, it would be reasonable to suspect some similar mechanism within turbulent vortices.

The scaling of turbulent vortex flows, however, has received relatively little attention compared to turbulent boundary layers. Based on dimensional arguments alone, Hoffman 
and Joubert ${ }^{15}$ demonstrated that turbulent vortices should be expected to exhibit a universal inner-scaled circulation profile of the form

$$
\begin{array}{ll}
\frac{\Gamma(\eta)}{\Gamma_{c}}=A_{0} \eta^{2} & 0 \leq \eta \lesssim 0.4 \\
\frac{\Gamma(\eta)}{\Gamma_{c}}=1+A_{1} \ln (\eta) & 0.5 \lesssim \eta \lesssim 1.4
\end{array}
$$

where $\Gamma$ is the circulation, $\eta=r / r_{c}$ is the nondimensional radial coordinate, and $r_{c}$ and $\Gamma_{c}$ are the vortex core radius and core circulation, respectively ( so that $\Gamma(1)=\Gamma_{c}$ ). By fitting (1) and (2) to experimental data, the constants $A_{0}$ and $A_{1}$ were found to be 1.83 and 0.929 , respectively. Alternatively, Phillips ${ }^{16}$ solved the Reynolds-averaged momentum equation subject to the usual line-vortex boundary conditions. A universal circulation profile emerged in the form of a polynomial in $\eta^{2}$, as

$$
\frac{\Gamma(\eta)}{\Gamma_{c}}=\sum_{k=1}^{n} B_{k} \eta^{2 k} \quad 0 \leq \eta \lesssim 1.3,
$$

where $B_{k}$ are constants. By a process of inner and outer matching, and requiring continuous velocities and velocity derivatives, unique values of $B_{k}$ emerged. The first three of these coefficients were found to be $B_{1}=1.7720, B_{2}=-1.0467$ and $B_{3}=0.2747$.

The Hoffman and Joubert ${ }^{15}$ and Phillips ${ }^{16}$ models agree well with each other, and there is a large amount of experimental data in support of them; see, for example, Ramaprian and Zheng ${ }^{17}$, Birch et al. ${ }^{18}$, and references cited therein. Moreover, the collapse has been shown to be remarkably robust, and insensitive to either free-stream turbulence (Bailey and Tavoularis ${ }^{19}$; see Figure 1) or unsteadiness ${ }^{10}$.

It is, however, interesting to note that laminar vortices also exhibit inner-scaled circulation profiles in close agreement with (1), (2) and (3). For example, the Batchelor ${ }^{20} q$-vortex, having a tangential velocity profile given by

$$
v_{\theta}(\eta)=v_{0}\left(1+\frac{1}{2 \alpha}\right) \frac{1}{\eta}\left(1-\exp \left(-\alpha \eta^{2}\right)\right),
$$

(where $v_{0}$ is the peak tangential velocity and $\alpha$ is defined by the transcendental $\mathrm{e}^{\alpha}=1+2 \alpha$, yielding $\alpha \approx 1.25643$ ), has a normalized circulation profile of

$$
\frac{\Gamma(\eta)}{\Gamma_{c}}=\frac{1-\exp \left(-\alpha \eta^{2}\right)}{1-\exp (-\alpha)},
$$

which can be expanded as

$$
\frac{\Gamma(\eta)}{\Gamma_{c}}=\sum_{i=k}^{n} \frac{(-1)^{k+1}}{k !} \frac{\alpha^{k}}{1-\exp (-\alpha)} \eta^{2 k} .
$$




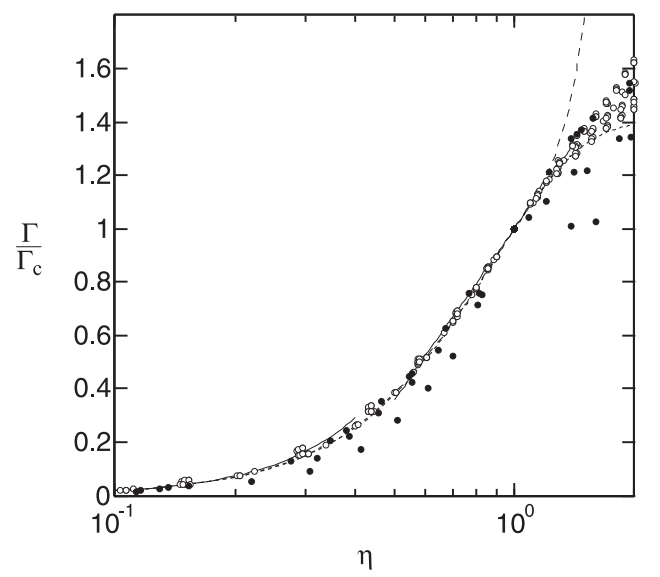

FIG. 1. Normalized circulation profiles. ○, Bailey and Tavoularis ${ }^{19}$; •, Phillips and Graham ${ }^{4}$; $\longrightarrow,(1) \&(2) ;---,(3) ;---,(5)$.

This series expansion yields coefficients of $1.7564,-1.1034$ and 0.4621 for $k=1,2$ and 3 , respectively, which are in close agreement with those found by Phillips ${ }^{16}$. One important implication of the above is the close approximation of (2) by (6); consequently, unlike the case of boundary layers, the existence of an apparent logarithmic region in the innerscaled circulation profile is not necessarily indicative of well-developed, scale-independent turbulence within a vortex.

\section{SIMILARITY OF TANGENTIAL VELOCITY PROFILES}

For the case of axisymmetric vortices, the circulation may be simply evaluated as the integral of tangential velocity around a circular path. The tangential velocity may therefore be expressed in terms of the circulation as

$$
\frac{v_{\theta}(\eta)}{v_{\theta}(1)}=\frac{1}{\eta} \frac{\Gamma(\eta)}{\Gamma_{c}}
$$

where $v_{\theta}(1)$ is the peak tangential velocity. Similarity of the circulation profiles therefore implies, for axisymmetric vortices, similarity of the tangential velocity profiles. The tangential velocity profiles, however, will be more sensitive to variations owing to the additional factor of $1 / \eta$. Substituting (3) into (7), then, yields a 'universal' tangential velocity profile of

$$
\frac{v_{\theta}(\eta)}{v_{\theta}(1)}=\sum_{k=1}^{n} B_{k} \eta^{2 k-1} \quad 0 \leq \eta \lesssim 1.3 .
$$


It should be noted that no constraints have yet been placed on the coefficients $B_{k}$. If $v_{\theta}$ is truncated at the fifth order (so that $B_{k}=0$ for $k \geq 4$ ), little precision is expected to be lost for $\eta \lesssim 1.2$ (see Phillips ${ }^{16}$ ). However, because $v_{\theta}(1)$ is, by definition, the local maximum of $v_{\theta}(\eta)$, the truncation then constrains $B_{2}$ and $B_{3}$ as

$$
\begin{aligned}
& B_{2}=\frac{5}{2}-2 B_{1} \\
& B_{3}=-\frac{3}{2}+B_{1},
\end{aligned}
$$

and $B_{1}$ (or the radial gradient of the tangential velocity at $\eta=0$ ) is restricted to values less than 1.875. Because (8) agrees well with several sets of experimental results ${ }^{10,17,19}$ as well as with (4), demonstrating collapse of the inner-scaled circulation distribution over $0 \leq \eta \lesssim 1.3$ is equivalent to demonstrating good agreement between the inner-scaled velocity profile and (8).

Tangential velocity profiles may similarly be obtained from Equations (1) and (2), as

$$
\begin{array}{ll}
\frac{v_{\theta}(\eta)}{v_{\theta}(1)}=A_{0} \eta & 0 \leq \eta \lesssim \eta_{i} \\
\frac{v_{\theta}(\eta)}{v_{\theta}(1)}=\frac{1}{\eta}+\frac{A_{1}}{\eta} \ln \eta & \eta_{i} \lesssim \eta \lesssim \eta_{o}
\end{array}
$$

where $\eta_{i}$ and $\eta_{o}$ are, respectively, the range of the inner and outer scaling. However, when expressed in this form, it becomes clear that (11) is, to some extent, inconsistent: if it is assumed that $\eta_{i} \lesssim 1 \lesssim \eta_{o}$ (and, of course, that $r_{c}$ is an appropriate inner length scale for a turbulent vortex), then (11) is required to have a local maximum at $\eta=1$. Therefore, the unique value $A_{1}=1$ is obtained. Using this value of the constant, a somewhat better agreement is obtained between (11) and experiment, though over a reduced range of $0.65 \lesssim$ $\eta \lesssim 1.2$ (Figure 2 ).

If it is further required that $v_{\theta}$ be continuous and differentiable over the domain $0 \leq$ $\eta \lesssim 1.3$ and that the buffer region around $\eta_{i}$ is small (the assumtions used in the analysis of Phillips ${ }^{16}$ ), unique values of $\eta_{i}$ and $A_{0}$ of 0.6065 and 1.3591, respectively, also emerge. However, using these values for the constants, (10) provides very poor agreement with experimental data for $\eta \lesssim \eta_{i}$. Ultimately, this only implies that the regions in which (10) and (11) are valid do not overlap. However, a closer examination of the data of Bailey and Tavoularis ${ }^{19}$ and Phillips and Graham ${ }^{4}$ suggests that collapse of the mean tangential velocity profiles is less robust in the region $0 \leq \eta<\eta_{i}$, especially for the cases of vortices formed in conditions of intense turbuelnt forcing (Figure 3). 
Self-similarity of trailing vortices

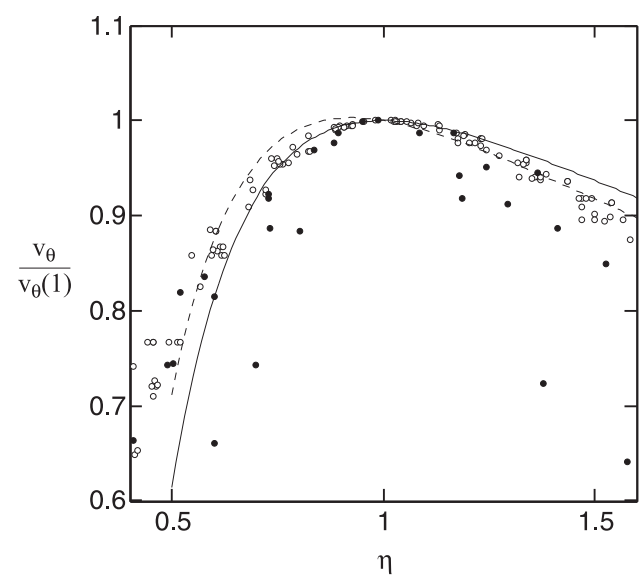

FIG. 2. Normalized tangential velocity profiles. o, Bailey and Tavoularis ${ }^{19}$; •, Phillips and Graham $^{4} ;---$, Hoffman and Joubert ${ }^{15} ;-,(11)$ with $A_{1}=1$.

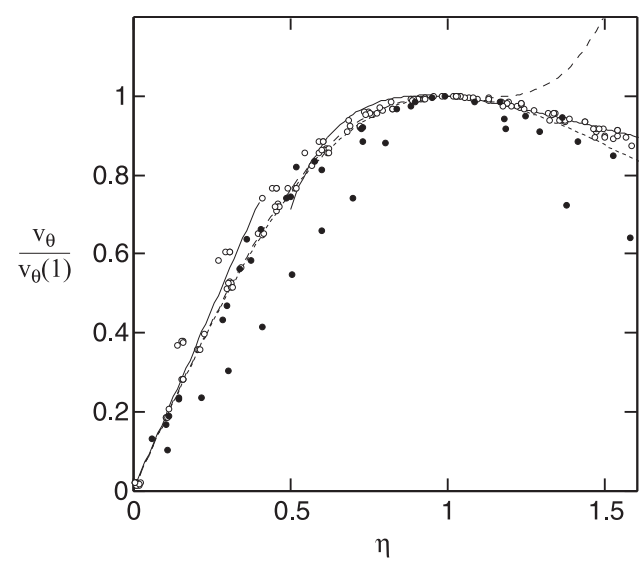

FIG. 3. Normalized tangential velocity profiles. ○, Bailey and Tavoularis ${ }^{19}$; •, Phillips and Gra$\operatorname{ham}^{4} ;-,(10) \&(11) ;---,(8) ;---,(4)$.

\section{A. Influence of vortex wandering}

Because the experimental measurement of vortex flows is often carried out using scanning velocity probes, the deconvolution of the velocity profiles to remove the effect of random 'wandering' has been the subject of a number of studies ${ }^{5,19,21,22}$. Typically, the effect of random wandering is assumed to impose a Gaussian distribution upon the location of the vortex centre (and therefore the origin of $\eta$ ), so that the 'apparent' velocity profile $v_{\theta}^{\prime}$ may 
be described as a convolution with a Gaussian kernel,

$$
v_{\theta}^{\prime}(x, y)=\frac{1}{2 \pi \sigma^{2}} \int_{-\infty}^{\infty} \int_{-\infty}^{\infty} \exp \left(-\frac{x_{0}^{2}+y_{0}^{2}}{2 \sigma^{2}}\right) v_{\theta}\left(x-x_{0}, y-y_{0}\right) d x_{0} d y_{0}
$$

where $\sigma$ is the standard deviation of the wandering amplitude (which is assumed isotropic), and the integral may be evaluated numerically with relative ease. Significantly, Monaghan and Humble ${ }^{23}$ demonstrated analytically that if (4) is substituted into (12) and integrated, the result is identical to (4), but with $v_{\theta}$ and $\eta$ re-scaled. Consequently, random wandering will have no effect on a vortex with a velocity profile described by (4) when scaled on inner parameters. Because the deconvolution of $v_{\theta}^{\prime}(\eta)$ requires an a priori assumption of the form of $v_{\theta}(\eta)$, a good agreement between $v_{\theta}^{\prime}(\eta)$ and (4) is usually taken as a necessary and sufficient condition that the deconvoluted $v_{\theta}(\eta)$ is of the form of (4) as well.

It is, however, important to recognize that for sufficiently large $\sigma$, the Gaussian convolution will drive any function $f(x, y)$ toward the Gaussian (an elegant proof of this is provided by Devenport et al. ${ }^{5}$ ). Consequently, in conditions of high-amplitude wandering (as will be the case for vortices formed in conditions of intense free-stream turbulence), the assumption that good agreement between $v_{\theta}^{\prime}$ and (4) implies good agreement between $v_{\theta}$ and (4) may no longer be valid, and the original form of the vortex may be lost.

To examine this effect, consider the axisymmetric, vortical flow which is well-described by the expression,

$$
\begin{aligned}
& \frac{v_{\theta}(\eta)}{v_{\theta}(1)}=\sum_{k=1}^{n} B_{k} \eta^{2 k-1} \quad \eta \leq \eta_{o} \\
& \frac{v_{\theta}(\eta)}{v_{\theta}(1)}=\frac{C}{\eta} \quad \eta>\eta_{o}
\end{aligned}
$$

where $\eta_{o}>1$ and $C$ is selected to ensure that $v_{\theta}$ is continuous. The inner region is given by (8), but the outer region is forced to vanish for large $\eta$, thereby ensuring (12) will remain finite and positive. If (13) is truncated to $k \leq 3$ and constrained by (9), the result is a tangential velocity profile which will closely ressemble (4) for $\eta \lesssim 1.2$ and $B_{1}=1.7720$, but which can easily be modified to represent very different possible rotating flows simply by varying $B_{1}$ (Figure 4$)$.

An error parameter $\phi$ is then defined, as

$$
\phi=\frac{\left|\int_{0}^{\eta_{o}} v_{\theta}^{*} d \eta-\int_{0}^{\eta_{o}} v_{\theta}^{\prime} d \eta\right|}{\int_{0}^{\eta_{o}} v_{\theta}^{*} d \eta}
$$


Self-similarity of trailing vortices

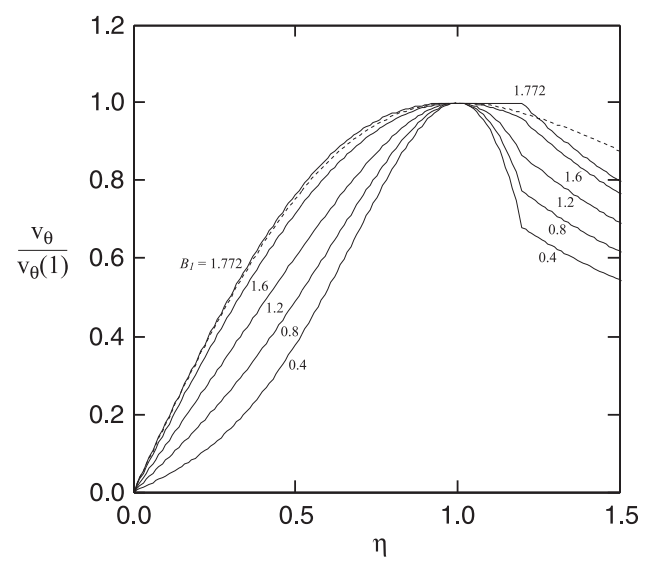

FIG. 4. Normalized tangential velocity profiles given by (14) truncated to the fifth order, with $\eta_{o}=1.2 .--,(4)$.

where $v_{\theta}^{*}$ is the tangential velocity profile of the nominal Batchelor $q$-vortex, given by (4). $\phi$ provides a measure of the error between the convoluted tangential velocity profile and the expected form. Figure 5 shows isocontours of $\phi$ as a function of $\sigma$ and $B_{1}$, for a range of rotating flows described by (13) and (14). For $\sigma / r_{c} \gtrsim 0.5, \phi$ is nearly insensitive to $B_{1}$, and for all $B_{1}$ in this range, $\phi \leq 0.02$, which is well within the level of error typical of experimental measurements.

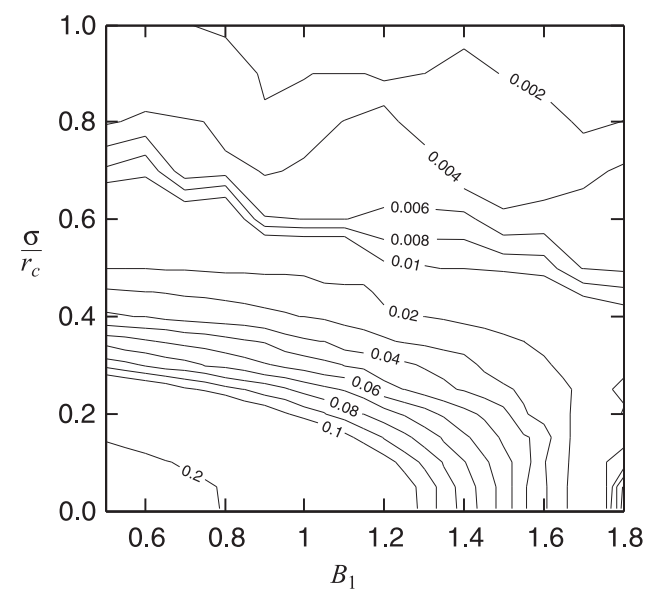

FIG. 5. Isocontours of the error parameter $\phi$ as a function of $\sigma$ and $B_{1}$, with $\eta_{o}=1.2$.

Because the magnitude of $\sigma$ required to cause $v_{\theta}^{\prime}$ to approximate $v_{\theta}^{*}$ decreases as $v_{\theta}$ approaches $v_{\theta}^{* 5}$, the validity of the assumption that the deconvoluted velocity profile is of the form of (4) may be assessed by applying the convolution (12) to a tangential velocity 
field which is highly non-Gaussian. As a limiting case, consider the discrete, axisymmetric tangential flow field described by

$$
\begin{array}{ll}
\frac{v_{\theta}(\eta)}{v_{\theta}(1)}=1 & 1-\epsilon \leq \eta \leq 1+\epsilon \\
\frac{v_{\theta}(\eta)}{v_{\theta}(1)}=0 & \eta<1-\epsilon \quad \& \quad \eta>1+\epsilon
\end{array}
$$

where $\epsilon$ is arbitrarily small. Though this flow field is clearly nonphysical, any real velocity field (having nonzero tangential velocity away from $\eta=1$ ) will begin to approximate (4) for smaller values of $\sigma$ when subjected to the Gaussian convolution. Figure 6 (a) shows the convoluted tangential velocity fields resulting from (16) for a range of $\sigma / r_{c}$. For $\sigma / r_{c} \gtrsim 0.8$,

the resultant $v_{\theta}^{\prime}$ approaches (4) to within typical experimental uncertainty. Furthermore, when the corresponding circulation is plotted on the usual semilogarithmic axes (Figure $6 \mathrm{~b}$ ), the curves are well-approximated by (5) for $\sigma / r_{c} \gtrsim 0.6$. Given that values of $\sigma / r_{c}$ exceeding 0.5 are not uncommon, especially within tip vortices far downstream of the generating wing or those produced in intense free-stream turbulence ${ }^{19}$, great care must be taken in implementing any deconvolution procedure in these circumstances.

\section{B. Influence of spatial averaging}

It is common in the analysis of experimental wake scans to infer the circulation profile by evaluating area integrals of vorticity and applying Green's theorem, so that

$$
\Gamma(r)=\iint_{A(r)} \omega(x, y) d A
$$

where $\omega$ is the streamwise component of vorticity and $A(r)$ is often taken as the area bounded by a circle of radius $r$. The resulting circulation estimate is then necessarily a spatial average. Consequently, it may be expected that a stationary system of discrete, similar co-rotating vortices will result in a circulation distribution approximating that of a Batchelor $q$-vortex if the vortices are distributed normally about the origin, and if there are a sufficiently large number of vortices in the system. However, this is unlikely to be of any practical significance, as the number of distinct co-rotating vortices commonly observed in tip-vortex and vortex generator experiments rarely exceeds 3 or $4^{18}$. On the other hand, it is possible that a discrete vortex sheet rolling up into a spiral around the origin may result in a normal radial distribution of circumferentially-averaged vorticity. Since incompletely-developed tip 

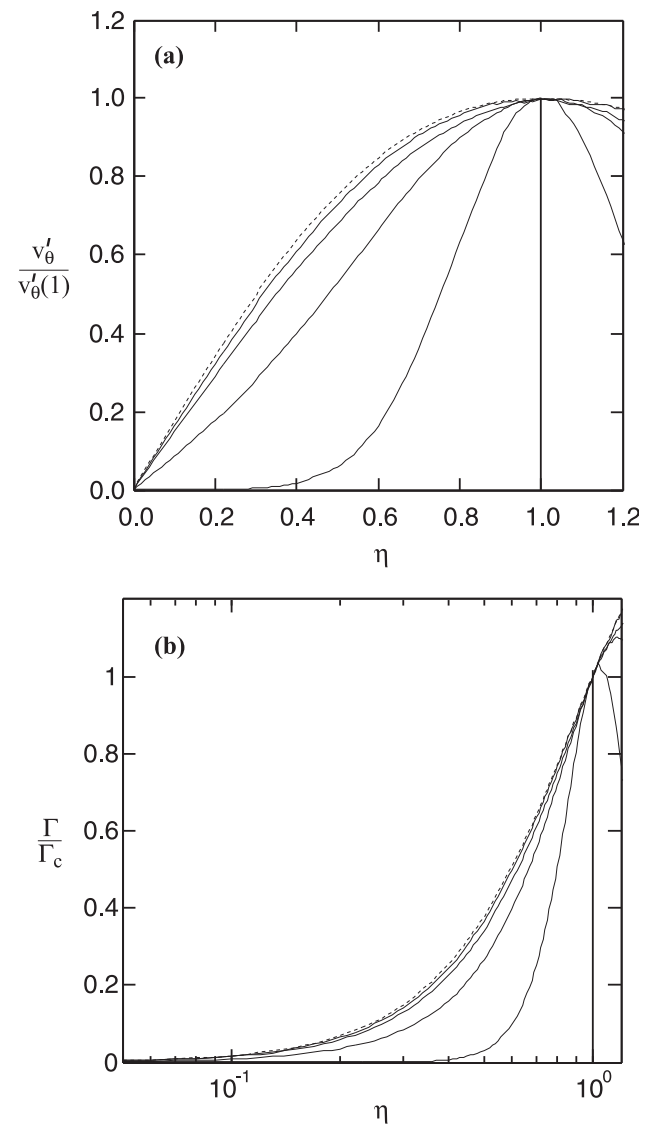

FIG. 6. Normalized velocity and circulation fields described by (16) subjected to random wandering with $\sigma / r_{c}=0,0.2,0.4,0.6$ and 0.8. (a) - - , (4); (b) - - , (5).

vortices may be represented in this way, the specific trajectory of the vortex sheet rolling up in the near-field of a wing should be considered further.

Moore and Saffman ${ }^{24}$ showed that, for the case of self-induced roll-up of an inviscid vortex sheet, the resulting tangenial velocity field is well-approximated by $v_{\theta} \propto 1 / \sqrt{r}$. This result agrees very poorly with experimental measurements, as viscous effects are not negligible in this flow. Devenport et al. ${ }^{5}$ provide experimental trajectories for a typical finite wing vortex sheet during roll-up, normalized against the position of the vortex centre $\xi$ (which varies as a function of downstream distance) and corrected for vortex wandering; these results are reproduced in Figure 7 (a). The spiral is very well approximated in polar coordinates by the expression,

$$
\rho(\theta) \propto \exp \left(k \theta^{2}\right)
$$


where $\rho$ is the radial position of the vortex sheet and $k \approx 0.028$ (Figure $7 \mathrm{~b}$ ). If the strength of the vortex sheet is assumed to be constant along its length (Devenport et al. ${ }^{5}$ show a variation in vorticity of $\sim 10 \%$ in the roll-up region, which also suggests that the wing loading was reasonably uniform) and the spiral is assumed to start at the origin, then the circulation will be proportional to the path length of the spiral (18), yielding,

$$
\Gamma(\eta)=g \int_{\theta_{i}}^{\theta}\left(1-2 \exp \left(k \theta_{0}^{2}\right)+\left(1+4 k^{2} \theta_{0}^{2}\right) \exp \left(2 k \theta_{0}^{2}\right)\right)^{1 / 2} d \theta_{0}
$$

where $g$ is a scaling factor, $\theta_{i}=0$ and $\theta=\sqrt{\ln (1+\eta) / k}$. Expanding (19) as a series and integrating yields, to the leading order, a normalized circulation profile of

$$
\frac{\Gamma(\eta)}{\Gamma_{c}}=\frac{\ln (1+\eta)}{\ln (2)}
$$

which is notably independent of $k$. When plotted in the usual semilogarithmic axes, (20) begins to closely approximate (2) for $\eta \gtrsim 0.8$ (Figure 8). Consequently, in at least some cases, it is possible that good agreement between a circulation profile obtained from experimental tip vortex wake scans and (2) at larger $\eta$ may be in part the result of the spatial averaging of discrete turns of the vortex sheet by the implementation of (17).

It is also very interesting to note that by carrying out the integration in (19) over a range which does not extend to the origin, it is possible to achieve much better agreement between the resulting circulation profile and that expected from a self-similar vortex. Practically, this flow could be equivalent to the very beginning of the roll-up process, prior to the formation of a well-developed core. Figure 9 compares the circulation profile obtained from (19) to the 'universal' circulation profile (5) for one particular case where $\theta_{i}>0$, and demonstrates that very good agreement is achieved over the range $0.4 \lesssim \eta \lesssim 1.1$. While this good agreement for particular values of $\theta_{i}$ may be spurious, the trajectory of the vortex sheet agrees well with the experimental measurements of Devenport et al. ${ }^{5}$ (Figure 9 inset), which was found to be independent of streamwise distance from the generating wing.

\section{CONCLUSIONS}

The circulation profiles of vortex flows have previously been shown to collapse remarkably well when scaled on core parameters. However, it is demonstrated that, for wandering amplitudes within the range of those observed experimentally, the Gaussian convolution 
Self-similarity of trailing vortices
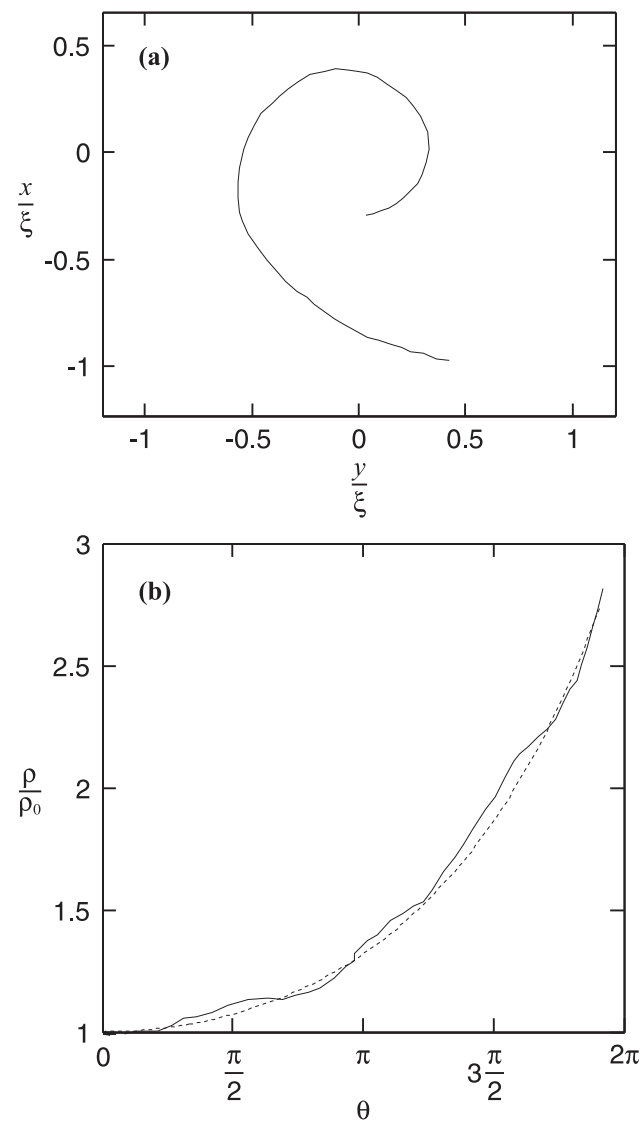

FIG. 7. Trajectory of vortex sheet during tip vortex roll-up (reproduced from Devenport et al. ${ }^{5}$ ), in (a) cartesian coordinates, and (b) polar coordinates. - - , (18); — origin is taken as the location of maximum streamwise vorticity.

resulting from the vortex wandering can cause any positive, axisymmetric flow field having zero velocity at $\eta=0$ and $\eta \rightarrow \infty$ to converge upon the 'universal' circulation profile obtained for the case of a Batchelor $q$-vortex. Furthermore, it has been demonstrated that a similar result may be obtained at larger $\eta$ for incompletely-developed wing-tip vortices. Care must therefore be taken in the implementation of any deconvolution procedure to remove the influence of vortex wandering, as a good agreement between the circumferentially-averaged mean tangential velocity field and the Batchelor $q$-vortex may not necessarily imply that the true vortex velocity profile also agrees well with the $q$-vortex. 
Self-similarity of trailing vortices

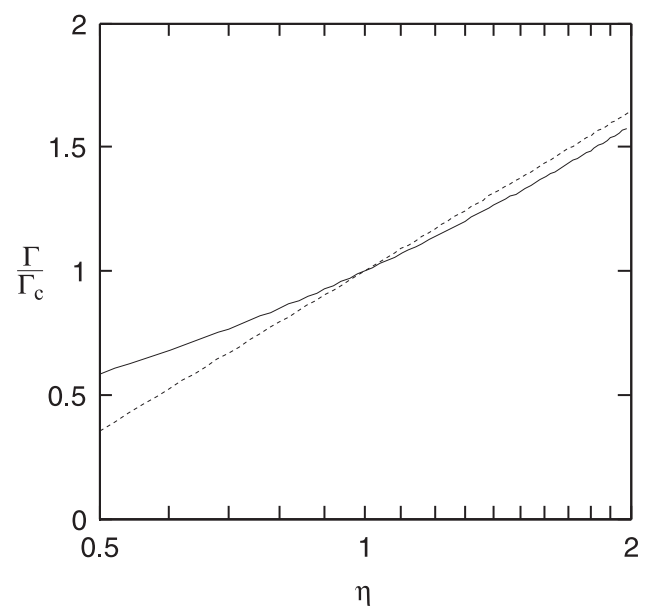

FIG. 8. Scaled circulation profile resulting from a vortex sheet rolling up with a trajectory described by $(18) .-,(19) ;---,(2)$.

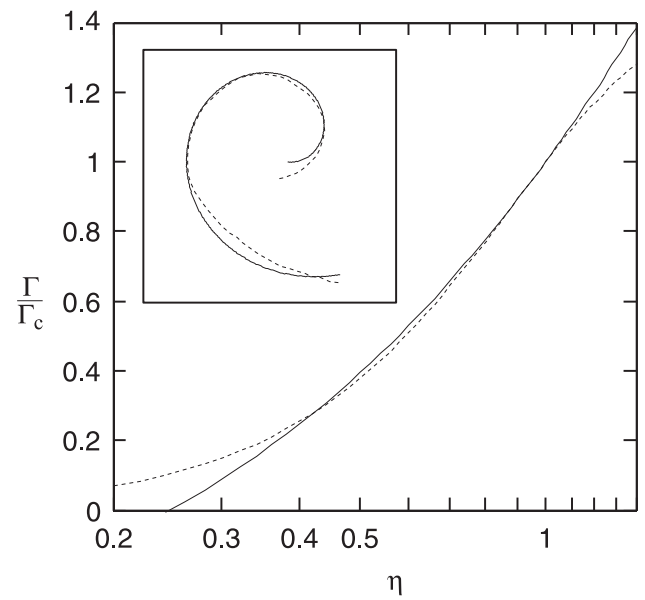

FIG. 9. Scaled circulation profile resulting from a vortex sheet rolling up with a trajectory described by (18), with $\theta_{i}>0$.,$-(19) ;---,(5)$. Inset: ——, trajectory of spiral over which integration has taken place; - - , re-scaled experimental results of Devenport et al. ${ }^{5}$.

\section{ACKNOWLEDGEMENTS}

This work was supported by the U.K. Engineering and Physical Sciences Research Council under grant number EP/H030360/1. The author is also grateful to Dr. Nick Hills for very helpful discussions. 


\section{REFERENCES}

${ }^{1}$ S. Widnall, "The structure and dynamics of vortex filaments," Ann. Rev. Fluid Mech. 7, 141-165 (1975).

${ }^{2}$ P. Spalart, "Airplane trailing vortices," Ann. Rev. Fluid Mech. 30, 107-138 (1998).

${ }^{3}$ I. Kroo, "Drag due to lift: concepts for prediction and reduction," Ann. Rev. Fluid Mech. 33, 587-617 (2001).

${ }^{4}$ W. R. C. Phillips and J. Graham, "Reynolds-stress measurements in a turbulent trailing vortex," J. Fluid Mech. 147, 353-371 (1984).

${ }^{5}$ W. J. Devenport, M. C. Rife, S. I. Liapis, and G. J. Follin, "The structure and development of a wing-tip vortex," J. Fluid Mech. 312, 67-106 (1996).

${ }^{6}$ J. Chow, G. Zilliac, , and P. Bradshaw, "Mean and turbulence measurements in the near field of a wingtip vortex," AIAA J. 35, 1561-1567 (1997).

${ }^{7}$ S. Green and A. Acosta, "Unsteady flow in trailing vortices," J. Fluid Mech. 227, 107-134 (1991).

${ }^{8}$ T. Sarpkaya, "Three-dimensional interactions of vortices with a free surface," AIAA paper 92-0059 (1992).

${ }^{9}$ M. Beninati and J. Marshall, "An experimental study of the effect of free-stream turbulence on a trailing vortex," Exp. Fluids 38, 244-257 (2005).

${ }^{10}$ D. M. Birch and T. Lee, "Investigation of the near-field tip vortex behind an oscillating wing," J. Fluid Mech. 544, 201-241 (2005).

${ }^{11}$ P. Bandyopadhyay, D. Stead, and R. Ash, "Organized nature of a turbulent trailing vortex," AIAA J. 29, 1627-1633 (1991).

${ }^{12} \mathrm{P}$. Bradshaw, "The analogy between streamline curvature and buoyancy in turbulent shear flow," J. Fluid Mech. 36, 177-191 (1969).

${ }^{13}$ A. Cotel and R. Breidenthal, "Turbulence inside a vortex," Phys. Fluids 11, 3026-3029 (1999).

${ }^{14}$ R. Mathis, N. Hutchins, and I. Marusic, "Large-scale amplitude modulation of the smallscale structures in turbulent boundary layers," J. Fluid Mech. 589, 147-156 (2004).

${ }^{15}$ E. R. Hoffman and P. N. Joubert, "Turbulent line vortices," J. Fluid Mech. 16, 395-411 (1963). 
${ }^{16}$ W. R. C. Phillips, "The turbulent trailing vortex during roll-up," J. Fluid Mech. 105, 451-467 (1981).

${ }^{17}$ B. R. Ramaprian and Y. Zheng, "Measurements in rollup region of the tip vortex from a rectangular wing," AIAA J. 35, 1837-1843 (1997).

${ }^{18}$ D. M. Birch, T. Lee, F. Mokhtarian, and F. Kafyeke, "Structure and induced drag of a tip vortex," J. Aircraft 41, 1138-1145 (2004).

${ }^{19}$ S. C. C. Bailey and S. Tavoularis, "Measurements of the velocity field of a wing-tip vortex, wandering in grid turbulence," J. Fluid Mech. 601, 281-315 (2008).

${ }^{20}$ G. K. Batchelor, "Axial flow in trailing line vortices," J. Fluid Mech. 20, 645-658 (1964).

${ }^{21}$ S. J. Beresh, J. F. Henfling, and R. W. Spillers, "Meander of a fin trailing vortex and the origin of its turbulence," Exp. Fluids 49, 599-611 (2010).

${ }^{22}$ G. V. Iungo, P. Skinner, and G. Buresti, "Correction of wandering smoothing effects on static measurements of a wing-tip vortex," Exp. Fluids 46, 435-452 (2009).

${ }^{23}$ J. J. Monaghan and R. J. Humble, "Vortex particle methods for periodic channel flow," J. Comput. Phys. 107, 152-159 (1993).

${ }^{24}$ D. W. Moore and P. G. Saffman, "Axial flow in laminar trailing vortices," Proc. R. Soc. Lond. A 333, 491-508 (1973). 